\title{
Manejo perioperatorio de la terapia antiplaquetaria dual en pacientes con implante reciente de stent coronario que requieren cirugía. Terapia puente
}

\author{
Perioperative management of dual antiplatelet therapy in recently \\ implanted coronary stent requiring surgery. Bridging therapy
}

Eder Meneses ${ }^{1}$, Carlos Fernández Pereira²

\section{RESUMEN}

Se realizan numerosas angioplastias que requieren tratamiento antiplaquetario, de los cuales 15-25\% necesitan alguna intervención quirúrgica luego del stent. Esto nos obliga a evaluar el riesgo hemorrágico del procedimiento quirúrgico y el riesgo trombótico antes de la interrupción de la terapia antiplaquetaria.

Se deben delimitar los tiempos de antiagregacion plaquetaria de acuerdo al tipo de stent usado y la urgencia de la cirugía, evaluar la posibilidad de diferir la cirugía hasta llevar a un tiempo seguro o de no ser posible diferir, evaluar la posibilidad de reemplazo por antiagregantes endovenosos.

Esta revisión realiza la evaluación de los riesgos isquémicos y hemorrágicos y la evidencia disponible en las opciones terapéuticas para un procedimiento frente a esta situación clínica.

Palabras claves: agentes antiplaquetarios, terapia puente, stents coronarios, perioperatorio, tirofibán.

\begin{abstract}
Several angioplasties are performed each year and require antiplatelet treatment; 15$25 \%$ of them need some surgical intervention after stent implantation.

We need to evaluate the hemorrhagic risk of the surgical procedure and the thrombotic risk before antiplatelet therapy interruption.

Platelet antiaggregation should be defined according to the type of stent used and the urgency of the surgery, evaluate the possibility of deferring the surgery until it takes a safe time or if it is not possible to defer, evaluate the possibility of replacement by endovenous antiaggregants.

This review evaluate ischemic and hemorrhagic risks and the evidence available in the therapeutic options for a procedure to manage this clinical situation.
\end{abstract}

Key words: antiplatelet agents, bridging therapy, cardiac stents, perioperative, tirofiban.

\section{INTRODUCCIÓN}

Actualmente se realizan numerosas intervenciones en pacientes con angioplastia e implantación de stent coronarios. $\mathrm{Al}$ mismo tiempo, requieren tratamiento antiplaquetario. De ellos, alrededor del 15-25\% necesitan algún tipo de procedimiento quirúrgico entre el primero y quinto año después de la implantación del stent ${ }^{1,9,12}$. Esta condición nos confronta con situaciones clínicas en las que tenemos que evaluar el riesgo hemorrágico del procedimiento quirúrgico y el riesgo trombótico antes de la interrupción de la terapia antiplaquetaria. Los pacientes que requieren cirugía después de una intervención coronaria percutánea tienen un mayor riesgo de infarto de miocardio y muerte cardíaca en comparación con los pacientes sin enfermedad arterial coronaria ${ }^{2.3}$. Por lo tanto, es importante conocer los riesgos de la cirugía y los riesgos trombóticos del stent. El propósito de esta revisión es evaluar los riesgos isquémicos y hemorrágicos y la evidencia disponible en las opciones terapéuticas para un procedimiento más racional frente a esta situación clínica.

1. Servicio de Cardiología Clínica. Sanatorio Otamendi. CABA

2. Servicio de Hemodinamia y Cardiología Intervencionista. Sanatorio Otamendi. CABA

$\triangle$ Correspondencia: Carlos Fernández Pereira MD, PhD, FACC. Centro de Estudios en Cardiología Intervencionista, Larrea 910, 4to piso A. C1117ABD CABA. Rep. Argentina. Tel/fax:+5491149629012. cfernandezpereira@centroceci.com.ar Los autores no declaran conflictos de intereses

Recibido: 02/09/2019| Aceptado: 30/09/2019

\section{MATERIAL Y MÉTODO}

Para la presente revisión se realizó una búsqueda sistemática en las bases de datos de la Biblioteca Nacional de Medicina - PubMed, American Heart Association, European Society of Cardiology, Journal of the American College of Cardiology, New England Journal of Medicine, centrada en la doble antiagregación después de la implantación del stent coronario y la duración de la doble antiagregación. Se utilizaron palabras clave como stent trombosis, terapia antiplaquetaria dual, stent de metal desnudo, stent liberador de fármacos. Luego se buscó el riesgo de hemorragia en pacientes con implante de stent que requieren cirugía, utilizando palabras clave como enfermedad arterial coronaria, cirugía cardíaca y cirugía no cardíaca. Finalmente, la búsqueda de opciones de tratamiento en pacientes con stent coronario y requerimiento de tratamiento quirúrgico se llevó a cabo con el término de búsqueda doble terapia antiplaquetaria y cirugía cardíaca y cirugía no cardíaca, y riesgo de sangrado y trombosis del stent y terapia puente. Se incluyeron estudios aleatorizados, controlados, doble ciego. seleccionados por orden de relevancia, así como los metaanálisis, se descartaron las pautas para la recomendación de las sociedades, el estado del arte, las series de casos y los informes de casos y series de casos publicados antes de 2001.

\section{EVOLUCIÓN DE LOS STENTS Y ANTIAGREGACIÓN PLAQUETARIA}

Los primeros stents en usarse fueron los metálicos o no farmacológicos, que disminuyeron en 20-30\% la oclusión aguda en comparación con la angioplastia con balón ${ }^{3}$. 
Esta técnica trajo consigo como efecto algunos casos de stent trombosis aguda con tasas de mortalidad de hasta el 8,9\% e incluso mayores en poblaciones especiales (diabéticos o insuficientes renales). Con el advenimiento de la terapia de doble antiagregación plaquetaria, la trombosis del stent disminuyó considerablemente con el uso de aspirina unida a clopidogrel por tiempo prolongado ${ }^{4}$ permitiendo la reendotelización del stent.

La aparición de reestenosis a causa de hiperplasia neointimal posterior al implante del stent hizo necesaria la reintervención durante el primer año posimplante del stent.

El advenimiento de los stents medicados de primera generación surgieron como la alternativa para solucionar el problema de la reestenosis; basados en la liberación de fármacos antineoplásicos o inmunosupresores desde su superficie, generan una inhibición de la proliferación del músculo liso y la hiperplasia endotelial, disminuyendo la incidencia de reestenosis ${ }^{3,6}$. Sin embargo, esta inhibición de la endotelización generaba un potencial trombogénico en la superficie metálica del stent. La suspensión prematura de la doble antiagregación plaquetaria evidenció la aparición de episodios de stent trombosis con cuadros de infarto agudo, arritmias malignas e incluso muerte súbita, con un riesgo aumentado en pacientes diabéticos, insuficientes renales o en stents implantados por lesiones en bifurcación $n^{7,8}$.

Actualmente, los stents liberadores de fármacos de última generación (zotarolimus o everolimus) representan el estándar de atención para la intervención coronaria percutánea de la enfermedad arterial coronaria. Los pacientes requieren tratamiento con doble antiagregación plaquetaria (aspirina + inhibidor p2y12, prasugrel, clopidogrel o ticagrelor) para evitar la stent trombosis hasta la endotelizacion del strut ${ }^{9}$.

Los tratamientos quirúrgicos son motivo frecuente de suspensión de la doble antiagregación plaquetaria para disminuir el riesgo de sangrado del acto quirúrgico, situación que, sumada al estado proinflamatorio y protrombótico generado por las cirugías, representa un riesgo importante de stent trombosis perioperatoria ${ }^{10}$.

Por otra parte, establecer el tiempo entre el implante de un stent y un tratamiento quirúrgico electivo es fundamental, ya que a menor tiempo transcurrido desde el implante de un stent y el momento en el cual se lleva a cirugía se asocia de forma independiente con muerte perioperatoria de origen cardíaco e infarto de miocardio, siendo el primer mes el de mayor riesgo independientemente del tipo de stent implantado ${ }^{11}$.

\section{RIESGO ISQUÉMICO. CONCEPTO DE RIESGO ISQUÉMICO COMBINADO}

Hay diversos factores que contribuyen a aumentar el riesgo de eventos cardíacos adversos en pacientes portadores de stents implantados recientemente y que van a ser sometidos a cirugía: la necesidad de doble antiagregación plaquetaria, el tiempo trascurrido desde el implante del stent hasta el momento de la cirugía, estados protrombóticos del peri- y posoperatorio, sumados a los factores de riesgo quirúrgicos y cardíacos propios de cada paciente juegan un papel fundamental en el riesgo isquémico.

Hay 2 variables que pueden ser modificables de ser necesario para disminuir los riesgos: una es el tiempo transcurrido desde la intervención coronaria percutánea hasta el momento de la cirugía y la otra es la suspensión de la doble an- tiagregación plaquetaria, variables sobre las cuales se pueden centrar los cambios necesarios si de disminuir riesgo isquémico se trata.

En la actualidad se habla de riesgo isquémico combinado para hacer referencia a estos factores ${ }^{12}$ los cuales analizaremos a continuación:

\section{TIEMPO DESDE LA INTERVENCIÓN CORONARIA PERCUTÁNEA HASTA LA CIRUGÍA}

El primer año posterior al implante de un stent, independientemente del tipo de stent usado, constituye un riesgo aumentado de eventos cardíacos y cerebrovasculares adversos en pacientes que son llevados a cirugía ${ }^{13}$.

Se plantea que, por un lado, a los pacientes que son llevados a cirugía se les realiza suspensión prematura de la doble antiagregación plaquetaria, situación que favorecería el aumento de eventos adversos; por otra parte, los efectos proinflamatorios y protrombóticos asociados a la cirugía, con activación de una respuesta inflamatoria sistémica, es otro factor que contribuye a aumentar el riesgo no solo de stent trombosis sino también de compromiso vascular coronario, ya que dicha respuesta genera mayor agregación plaquetaria y menor respuesta a la terapia antiplaquetaria.

Se analizaron muestras de sangre de pacientes en posoperatorio de cirugía vascular mayor y encontraron que la expresión de diversas sustancias generaban un incremento en la activación plaquetaria y que había una asociación directa con los pacientes que presentaban elevación en los niveles de troponina; así, se evidenciaron niveles elevados de P-selectina y enlaces de fibrinógeno significativamente mayores en los pacientes que presentaron niveles elevados de troponina I, con la consecuente mayor activación de la agregación plaquetaria y menor respuesta a la aspirina en comparación con los pacientes que no tenían niveles elevados de troponina $\mathrm{I}^{10}$. El momento ideal para la realización de una cirugía luego del implante de un stent coronario aún es tema de debate. A la fecha no se dispone de estudios aleatorizados que guíen dicha conducta.

Las recomendaciones sugeridas por las sociedades para las cirugías que requieren la suspensión del inhibidor P2Y12 son las siguientes:

La Sociedad Europea de Cardiología recomienda diferir la cirugía hasta pasados por lo menos 1 mes independientemente del tipo de stent, siempre y cuando la aspirina pueda ser mantenida durante el período perioperatorio. Recomendación clase IIa, nivel de evidencia B. Para los pacientes con infarto de miocardio reciente u otras características de alto riesgo de isquemia que requieran doble antiagregación plaquetaria, la cirugía electiva debe posponerse por al menos 6 meses $^{14}$.

Por su parte, la American Heart Association desaconseja la cirugía no cardíaca dentro de los 3 meses posteriores al implante de un stent liberador de fármacos; la cirugía podría ser considerada entre los 3 a 6 meses luego del implante de un stent farmacológico con interrupción de la doble antiagregación plaquetaria si el riesgo quirúrgico es mayor que el riesgo de stent trombosis, aunque se considera óptimo luego de pasados los 6 meses del implante del stent. En el caso de los stents convencionales, recomiendan retrasar la cirugía no cardíaca al menos 1 mes después de implantado el stent ${ }^{9}$. 


\section{SUSPENSIÓN PREMATURA DE LA DOBLE ANTIAGREGACIÓN PLAQUETARIA}

Las complicaciones trombóticas relacionadas con el stent se atenúan mediante el uso de doble antiagregación plaquetaria, lo cual permite la endotelizacion, proceso que puede durar varios meses ${ }^{15}$.

La suspensión prematura de la doble antiagregación plaquetaria durante este período se asocia a aumento del riesgo de eventos isquémicos ${ }^{8,16}$.

Las recomendaciones de la Sociedad Europea de Cardiología y de la American Heart Association sugieren el uso de doble antiagregación plaquetaria por 6 meses para los pacientes con enfermedad arterial coronaria estable que recibieron el implante de un stent farmacológico de segunda generación $^{8,13}$, aunque, dependiendo del stent usado, el riesgo isquémico y el riesgo de sangrado, podría considerarse, de ser necesaria, la suspensión prematura de la doble antiagregación plaquetaria $^{9,14,17}$.

\section{CARACTERÍSTICAS CLÍNICAS Y ANGIOGRÁFICAS}

Hay diversos factores y características clínicas que pueden ser predictores de un evento cardíaco adverso mayor en el perioperatorio. El antecedente de síndrome coronario agudo en el último año, diabetes mellitus, insuficiencia renal, insuficiencia cardíaca congestiva o el deterioro de la fracción de eyección del ventrículo izquierdo son predictores importantes de evento cardíaco adverso mayor ${ }^{12,18,19}$.

Por otro lado, las características angiográficas tales como la presencia de stents largos o múltiples (al menos 3 stents implantados o 3 lesiones tratadas o longitud total del stent $>60$ $\mathrm{mm})$, stents superpuestos, diámetro del stent $<2,5 \mathrm{~mm}$, lesiones en bifurcación, enfermedad coronaria extensa o revascularización incompleta, pueden diferenciar a los pacientes que se beneficiarían con terapia antiplaquetaria dual por tiempo prolongado, ya que estos hallazgos podrían vincularse con mayor extensión de la aterosclerosis y la necesidad de revascularización compleja con mayor riesgo de eventos isquémicos tempranos y tardíos ${ }^{12,20,21}$.

\section{TIPO DE STENT}

Es difícil establecer un mejor perfil de stents farmacológicos de última generación comparados con los no farmacológicos de cromo-cobalto. Un estudio realizado en Ontario informó que el tiempo óptimo de espera para la realización de una cirugía era de 46 a 180 días para stents no farmacológicos y de 180 días para stents liberadores de fárma$\cos ^{22}$. En el estudio comparativo ORAR $\mathrm{II}^{45}$ los dos grupos de BMS + rapamicina oral (RO) y el grupo BMS solo recibieron 30 días de clopidogrel, observándose una significativa reducción de la reestenosis en el grupo RO, pero además que en ambos grupos se podían realizar intervenciones quirúrgicas sin dificultad a partir de los 30 días. En el estudio ORAR III ${ }^{46}, 200$ pacientes fueron aleatorizados a BMS + RO vs. DES. La RO fue administrada como bolo de $10 \mathrm{mg}$ vía oral antes de la angioplastia y luego $3 \mathrm{mg} \mathrm{du}$ rante 13 días. A 18,3 \pm 7 meses de seguimiento la estrategia de RO presentó un mejor costo-beneficio en forma significativa comparado con los DES $(p=0,0001)$, sin diferencias significativas en la revascularización del vaso tratado y en la necesidad de nueva revascularización de la lesión tratada. Por otra parte, en este estudio la gran ventaja fue en el grupo de BMS con respecto a necesidad de cirugía de urgencia a partir de los 30 días en los cuales los pacientes se encontraban sin clopidogrel y por consiguiente no tenían contraindicación a las intervenciones quirúrgicas las cuales no se podían establecer el tratamiento de la misma forma en el grupo DES.

En otro estudio retrospectivo los factores más fuertemente asociados con los eventos adversos mayores tuvieron que ver con la admisión quirúrgica no selectiva y el antecedente de infarto de miocardio reciente, pero no con el tipo de stent cuando el momento de la cirugía fue más de 6 meses después del implante del stent. La mayor tasa de eventos cardíacos adversos sucedieron entre el primero y tercer mes .Pero en este caso los resultados podrían estar sesgados en la decisión de implantar stents convencionales en pacientes con mayor deterioro y riesgo clínico ${ }^{23}$.

\section{EFECTOS DE LA DOBLE ANTIAGREGACIÓN PLAQUETARIA Y EL SANGRADO PERIOPERATORIO}

En este punto resulta de suma importancia establecer el riesgo hemorrágico como la otra parte del análisis en lo relacionado al manejo perioperatorio de la doble antiagregación plaquetaria en pacientes con implante reciente de stent coronario que van a ser sometidos a una intervención quirúrgica. La estratificación se debe individualizar respecto del tipo de procedimiento quirúrgico específico y no solo del papel que ejerce la doble antiagregación en el riesgo hemorrágico.

Una revisión sistemática demostró que la continuación perioperatoria de la aspirina tiene un efecto estadísticamente significativo (pero clínicamente modesto) sobre el riesgo de sangrado después de cirugía de revascularización miocárdica ${ }^{25}$; el sangrado aumenta con dosis de aspirina $>325 \mathrm{mg}$ al día, pero no con dosis más bajas. Por otra parte, en cirugía no cardíaca, el uso de aspirina perioperatoria no representó un aumento en el riesgo de hemorragia entre los pacientes que recibieron aspirina $75 \mathrm{mg} \mathrm{du}-$ rante todo el período perioperatorio. Sin embargo, hay un mayor riesgo de hemorragia en pacientes que son sometidos a ciertos tipos de cirugía, especialmente cirugía intracraneal, artroplastia de cadera, amigdalectomía y prostatectomía transuretral ${ }^{24,26}$.

Por otra parte, el uso combinado de aspirina con un inhibidor P2Y12 representa mayor riesgo de sangrado, transfusiones y días de internación en terapia intensiva ${ }^{27}$.

En una revisión se evaluaron pérdidas sanguíneas, aumentando entre $2,5-20 \%$ por aspirina y $30-50 \%$ por clopidogrel, la necesidad de transfusiones aumentó $30 \%$, pero la mortalidad no aumentó, excepto en cirugía intracraneana y resección transuretral de próstata ${ }^{28}$.

El riesgo hemorrágico se debe establecer clasificando el procedimiento quirúrgico a realizar, así el riesgo bajo lo constituyen las intervenciones en que la hemostasia se puede conseguir adecuadamente, una posible hemorragia no supone un riesgo vital para el paciente ni compromete el resultado de la cirugía y no requiere transfusión.

El riesgo hemorrágico moderado define procedimientos en los que la hemostasia quirúrgica puede ser difícil y la hemorragia aumenta la necesidad de transfusión o reintervención. 
Por último, el riesgo hemorrágico alto se ha definido como aquel en que la hemorragia perioperatoria puede comprometer la vida del paciente o el resultado de la cirugía ${ }^{29}$.

Además de los riesgos hemorrágicos dados por la doble antiagregación plaquetaria y por las características de cada cirugía, se deben tener en cuenta las comorbilidades de cada paciente en particular así como los procedimientos en los que pueden ser catalogados de bajo riesgo pero que por no tener fácil acceso a hemostasia pueden ser considerados de un riesgo mayor.

\section{RECOMENDACIONES PARA EL MANEJO PERIOPERATORIO DE LA DOBLE ANTIAGREGACIÓN PLAQUETARIA}

El tratamiento antiplaquetario perioperatorio debe definirse teniendo en cuenta tanto el riesgo isquémico y/o trombótico de cada paciente como el riesgo hemorrágico relacionado con el procedimiento quirúrgico.

El riesgo trombótico corresponde a la necesidad esencial de mantener la doble antiagregación plaquetaria, contempla el tiempo desde la intervención coronaria percutánea hasta la cirugía, las características clínicas, angiográficas y el tipo de stent, y se clasifica en bajo, intermedio y alto riesgo trombótico $^{10}$ (Tablas 1 y 2).

En los pacientes sometidos a cirugía precozmente después de la intervención coronaria percutánea (dentro de 1 mes y hasta 3 meses), todavía hay un mayor riesgo trombótico incluso si se mantiene la terapia antiplaquetaria dual ${ }^{30}$. Se recomienda remitir a los pacientes de alto riesgo a centros donde estén disponibles las técnicas quirúrgicas mínimamente invasivas y donde se disponga de hemodinamia permanente ${ }^{12,14}$.

\section{CIRUGÍA ELECTIVA}

Un análisis post hoc del ensayo POISE-2 (Perioperative Ischemic Evaluation-2) de pacientes con antecedentes de intervención coronaria percutánea mostró que la aspirina redujo el riesgo del compuesto de muerte o infarto de miocardio en un $50 \%$ comparada con placebo independientemente del tipo de stent y el momento del implante ${ }^{31}$.

Estos hallazgos respaldan el beneficio de continuar el uso de aspirina a bajas dosis durante el período perioperatorio en pacientes con intervención coronaria percutánea previa a la cirugía no cardíaca.

La gran mayoría de los procedimientos se pueden realizar con aspirina ${ }^{26,32}$, con la posible excepción de las cirugías con un riesgo extremadamente alto de hemorragia tales como neurocirugía, cirugía de canal medular, prostatectomía transuretral, procedimientos intraoculares de cámara posterior, hepatectomía o duodenopancreatectomía ${ }^{12,14}$.

Respecto a la terapia con inhibidores P2Y12 se ha demostrado que la interrupción 3 días antes de la cirugía, en comparación con 5 días, no aumentó la incidencia de complicaciones hemorrágicas mayores con ticagrelor, pero sí con clopidogrel $^{33}$. El ensayo TRITON-TIMI encontró que la tasa de sangrado relacionado con cirugía de revascularización miocárdica fue cuatro veces mayor en los pacientes que recibieron el bloqueador del receptor de ADP irreversible prasugrel que en los que recibieron clopidogrel ${ }^{34}$.

Sobre la base de estos hallazgos, las recomendaciones para la suspensión de los inhibidores del receptor P2Y12 suge- ridas por la Sociedad Europea de Cardiología en pacientes que necesitan ser llevados a cirugía son, suspender el uso de ticagrelor 3 días previo a la cirugía, para el clopidogrel se sugiere suspender 5 días previo a la cirugía y para el prasugrel 7 días previo a la cirugía en pacientes con intervención coronaria percutánea reciente (1-3 meses antes de la cirugía) $)^{14}$.

En los pacientes que presentan un alto riesgo de trombosis $(<1$ mes de intervención coronaria percutánea) y que requieran ser sometidos a cirugía que no se puede diferir y con alto riesgo de hemorragia, la terapia puente con antiplaquetarios endovenosos como el tirofibán puede ser una opción factible $^{12,14}$.

\section{CIRUGÍA URGENTE}

Se define como la cirugía requerida dentro de las siguientes 48 horas. En este tipo de pacientes se debe realizar una evaluación multidisciplinaria acerca del momento de la cirugía junto con los cirujanos y anestesiólogos, el riesgo y los beneficios.

En caso de procedimientos quirúrgicos urgentes considerados de alto riesgo hemorrágico, la terapia antiplaquetaria debe interrumpirse inmediatamente e implementarse las posibles medidas de soporte perioperatorio en caso de exceso de hemorragia.

A la fecha no hay antídotos en uso cotidiano para los agentes antiplaquetarios, con lo cual el único medio posible para la recuperación de la función plaquetaria es la transfusión de plaquetas. Idealmente, las transfusiones de plaquetas se deben administrar después de que el agente antiplaquetario oral ya no esté en circulación para evitar que las plaquetas recién transfundidas se inhiban.Para la aspirina, la vida media es de 15 a 20 minutos; para inhibidores orales del receptor P2Y12 hasta 6 horas (más tiempo para prasugrel que para clopidogrel); la vida media del ticagrelor y su principal metabolito es de aproximadamente 10 a 12 horas.

Por lo tanto, las transfusiones de plaquetas deben evitarse dentro de las 4 a 6 horas después de la última dosis de una tienopiridina y de 10 a 12 horas después de la última dosis de ticagrelor $^{12,14,17}$

\section{TERAPIA PUENTE CON ANTIPLAQUETARIOS ENDOVENOSOS}

La terapia puente constituye una opción terapéutica transitoria con drogas antiplaquetarias endovenosas en pacientes que requieren doble antiagregación plaquetaria y no pueden recibir tratamiento con antiplaquetarios por vía oral.

Se reserva generalmente para pacientes con alto riesgo trombótico que no pueden interrumpir el tratamiento antiplaquetario vía oral, que van a ser sometidos a cirugía no diferible con alto riesgo hemorrágico, en los cuales se requiere una interrupción predecible de la inhibición plaquetaria al momento de la cirugía ${ }^{9,12,36}$.

Tanto en las guías actuales de la Sociedad Europea de Cardiología como en las guías de la American Heart Association se plantea la terapia puente con antiplaquetarios endovenosos como una estrategia de tratamiento a considerar cuando se deben suspender los antiplaquetarios orales ${ }^{9,14}$.

Los agentes antiplaquetarios endovenosos disponibles son el cangrelor y los inhibidores de la glicoproteína IIb-IIIa: tirofibán y eptifibatide ${ }^{12}$. 


\section{Cangrelor}

Cangrelor es un inhibidor del receptor P2Y12 endovenoso, no se elimina por vía renal por lo que no requiere ajuste de dosis en pacientes con insuficiencia renal, tiene unión reversible a los receptores P2Y12 y una vida media corta, 3 a 6 minutos, características que permiten una activación de la función plaquetaria aproximadamente 60 minutos después de su suspensión ${ }^{37,38}$.

El uso de cangrelor redujo significativamente la tasa de eventos isquémicos incluyendo stent trombosis en intervención coronaria percutánea sin aumento significativo en el riesgo de sangrado ${ }^{39}$.

Sin embargo, no hay estudios aleatorizados controlados de terapia puente con cangrelor en cirugía no cardíaca.

Por otra parte, en cirugía cardíaca, el estudio BRIDGE, un estudio prospectivo, randomizado, doble ciego, comparado con placebo y multicéntrico, evaluó el uso de cangrelor contra placebo como terapia puente en pacientes con síndrome coronario agudo o tratados con implante de stent que iban a ser llevados a cirugía de revascularización miocárdica, resultando en una gran tasa de mantenimiento de inhibición plaquetaria sin diferencias significativas en sangrado mayor, aunque con episodios significativos de sangrado menor ${ }^{40}$.

Siguiendo el enfoque del estudio BRIDGE, el uso de cangrelor en dosis de $0,75 \mu \mathrm{g} / \mathrm{kg} / \mathrm{min}$ sin dosis de carga, sin ajustar a la función renal, se puede iniciar como terapia puente, comenzando al momento de la siguiente dosis esperada del inhibidor P2Y12 vía oral y se puede suspender hasta 1 hora antes del inicio de la cirugía.

Debido a que los niveles de agentes antiplaquetarios orales persisten luego de su suspensión, es razonable esperar 72 horas para el inicio de cangrelor ${ }^{12,36,41}$ (Figura 2).

\section{Inhibidores de la glicoproteína IIb-IIla}

Por otro parte, los inhibidores de la glicoproteína IIb-IIIa eptifibatide o tirofibán también pueden ser considerados como alternativa para la terapia puente ${ }^{9,14}$.

Sin embargo, a diferencia del cangrelor, tienen una vida media más larga, se eliminan por vía renal y requieren ajuste de dosis en pacientes con insuficiencia renal

No hay estudios con inhibidores de glicoproteína IIb-IIIa que hayan evaluado un régimen de terapia puente. Los datos derivan de estudios observacionales, que usan similar dosis de mantenimiento empleada para síndrome coronario agudo luego de intervención coronaria percutánea.

Por ende, el riesgo de complicaciones hemorrágicas puede llegar a ser mayor debido al mayor tiempo de infusión que conduce a una supresión casi completa de la función plaquetaria ${ }^{42,43}$.

Se recomienda la suspensión de inhibidores del receptor P2Y12 vía oral (prasugrel, 7 días antes de la cirugía; clopidogrel, 5 dias; y ticagrelor, 3 dias) e inciar la infusión con tirofibán a dosis de carga de $0,4 \mu \mathrm{g} / \mathrm{kg} / \mathrm{min}$ por 30 minutos, seguida de dosis de mantenimiento a razón de $0,1 \mu \mathrm{g} / \mathrm{kg} / \mathrm{min}$, o eptifibatide $(2 \mu \mathrm{g} / \mathrm{kg} / \mathrm{min}) 3$ días antes de la intervención quirúrgica; debe interrumpirse 4 a 6 horas antes de la cirugía ( 8 horas antes en pacientes con clearance de creatinina $<50$ $\mathrm{ml} / \mathrm{min}$ y a dosis ajustada, para tirofiban $0,05 \mu \mathrm{g} / \mathrm{kg} / \mathrm{min}$, para eptifibatide $1 \mu \mathrm{g} / \mathrm{kg} / \mathrm{min})^{36,42,43,44}$ (Figura 1).

Una vez lograda la hemostasia, la terapia antiagregante dual vía oral debe iniciarse dentro de las siguientes 24 a 48 horas. En pacientes con mayor riesgo de hemorragia, se sugiere rotar el tratamiento a clopidogrel. Si no es posible reiniciar la vía oral por alguna condición clínica, la infusión endovenosa con antiagreantes plaquetarios (cangrelor, tirofibán o eptifibatide) debe ser reiniciada después de la cirugía evaluando previamente el riesgo de sangrado.

Si es posible reiniciar con inhibidores P2Y12 por vía oral, se debe realizar una dosis de carga. Como se mencionó previamente, las complicaciones trombóticas se presentan con mayor frecuencia en el posoperatorio debido a estados protrombóticos, por lo que se recomienda monitoreo continuo en unidad cerrada ${ }^{12}$.

\section{DISCUSIÓN}

Se recomienda terapia antiplaquetaria dual durante al menos 1 mes después del implante de un stent no farmacológico, durante 6 meses después del implante de un stent farmacológico de nueva generación, y hasta 1 año en pacientes después de un síndrome coronario agudo independientemente de la estrategia de revascularización.

Por ende, el riesgo de complicaciones hemorrágicas puede llegar a ser mayor debido al mayor tiempo de infusión que conduce a una supresión casi completa de la función plaquetaria ${ }^{42,43}$. Actualmente, con los stents de nueva generación, podría ser aceptable disminuir los tiempos de antiagregación plaquetaria dual mínima necesaria a 1 mes (stent no farmacológico) o a 3 meses (stent farmacológico de nueva generación), independientemente de las características agudas de la enfermedad coronaria, en los casos en que la cirugía no se puede retrasar. Sin embargo, tal procedimiento quirúrgico debe ser realizado en hospitales donde haya disponibilidad de hemodinamia 24 horas todos los días de la semana ${ }^{17}$

Se debe valorar la necesidad de intervención electiva mientras el riesgo trombótico sea moderado-alto.

De ser posible, posponer la intervención quirúrgica hasta que el riesgo trombótico se considere bajo.

De no ser posible el hecho de posponer la cirugía, se recomienda mantener la aspirina excepto en procedimientos que lo contraindiquen (neurocirugía, cirugía de canal medular, prostatectomía transuretral, procedimientos intraoculares de cámara posterior, hepatectomía o duodenopancreatectomía) ${ }^{12,14}$.

Si el riesgo trombótico es moderado, se debe suspender el inhibidor del receptor P2Y12 antes de la cirugía, excepto en situaciones de bajo riesgo hemorrágico en los cuales se podría mantener; se sugiere suspender el ticagrelor 3 días previo a la cirugía, el clopidogrel 5 días previo a la cirugía y el prasugrel 7 días previo a la cirugía ${ }^{12,17,29}$.

En cirugía urgente, en caso de alto riesgo trombótico, la decisión depende del riesgo hemorrágico de la intervención quirúrgica; si el riesgo hemorrágico es bajo se recomienda no suspender la doble antiagregación plaquetaria; si el riesgo hemorrágico es moderado a grave se recomienda individualizar cada caso y tomar la decisión con equipo multidisciplinario; en pacientes con alto riesgo hemorrágico y alto riesgo trombótico, la terapia puente con antiplaquetarios endovenosos puede ser considerada ${ }^{12,17,29}$.(Anexo 1).

\section{CONCLUSIONES}

Resulta fundamental una adecuada estratificacion previa a la realización de un procedimiento quirúrgico en el contexto de pacientes con implante reciente de stents coronarios, ya que establecer el riesgo-beneficio perioperatorio nos va a per- 
mitir minimizar los riesgos tanto hemorrágicos posoperatorios como el riesgo de stent trombosis.

Se deben delimitar los tiempos de antiagregación plaquetaria con base en los tipos de stent usados previamente y la urgencia de intervención quirúrgica para evaluar la seguridad de la suspensión de la doble antiagregacion, la posibilidad de diferir la cirugía hasta llevar a un tiempo seguro para la suspensión de los antiagregantes plaquetarios o de no ser posible diferir la cirugía, evaluar la posibilidad de reemplazo por antiagregantes endovenosos.

La conducta definitiva debería realizarse basada en un enfoque interdisciplinario con un equipo médico conformado idealmente por cardiólogos, cirujanos, anestesistas y hematólogos.
Por lo general y salvo excepciones, se puede mantener el tratamiento con aspirina, pero los inhibidores P2Y12 generalmente deben ser suspendidos excepto en cirugías de bajo riesgo hemorrágico.

En situaciones clínicas de alto riesgo trombótico y alto riesgo hemorrágico la terapia puente es una opción a considerar, demostrada en otros contextos clínicos con perfiles de eficacia y de seguridad adecuados.

Se requieren estudios en cirugía no cardíaca que permitan establecer eficacia y seguridad de la terapia puente, ya que hasta el momento se dispone de series de casos con evidencia limitada y poco peso estadístico, resultando un campo interesante para futuras investigaciones.

\section{BIBLIOGRAFÍA}

1. Benjamin EJ, Blaha MJ, Chiuve SE, et al., American Heart Association Statistics Committee and Stroke Statistics Subcommittee. Heart Disease and Stroke Statistics-2017 Update: a report from the American Heart Association. Circulation 2017; 135:e146-603.

2. Rodríguez A, Palacios I, Fernández M, Larribau M, Giraudo M, Ambrose J. Time course and mechanism of early luminal diameter loss after percutaneous transluminal coronary angioplasty December 1, 1995Volume 76, Issue 16, Pages 1131-1134. The American Journal of Cardiology

3. Serruys PW, KutrykMJ, Ong AT, Metzler H, James SK. Coronary-arterystents. NEnglJMed 2006; 354: 483-95.

4. MehtaSR, YusufS, PetersRJG, et al. Effects of pretreatment with clopidogrel and aspirin followed by long-term therapy in patients undergoing percutaneous coronary intervention: the PCI-CURE, for the Clopidogrel in Unstable angina to prevent Recurrent Events trial (CURE) Investigators study.

5. Cuttip DF, Balm DS, HoK, et al. Stent thrombosis in the modern era: analisis ofmulticenter coronary stent clinical trials. Circulation 2001; 103: 1967-71.

6. Stone GW, Moses JW, Ellis S, et al. Safety and efficacy of sirolimus and paclitaxel eluting coronary stents. N Engl J Med 2007; 356: 998-1008.

7. Rodríguez AE, Mieres J, Fernández-Pereira C, Jiménez A. Coronary stent thrombosis in the current drug-eluting stent era: insights from the ERACl III trial. JAmCollCardiol 2006; 47: 205-7

8. lakovou I, Schmidt T, Bonizzoni E, et al. Incidence, predictors, and outcome of thrombosis after successful implantation of drug-eluting stents. JAMA 2005: 293: 2126-30

9. Levine GN, Bates ER, Bittl JA, et al. 2016 ACC/ AHA guideline focused update on duration of dual antiplatelet therapy in patients with coronary artery disease: a report of the American College of Cardiology/American Heart Association Task Force on Clinical Practice Guidelines. J Am CollCardiol 2016:68:1082-115.

10. Rajagopalan S, FordI, Bachoo P, et al. Platelet activation, myocardial ischemic events and postoperative non-response to aspirin in patients undergoing major vascular surgery. J ThrombHaemost 2007:5:2028-35

11. Saia F, Belotti LM, Guastaroba P, et al. Risk of adverse cardiac and bleeding events following cardiac and noncardiac surgery in patients with coronary stent: how important is the interplay between stent type and time from stenting to surgery? CircCardiovascQualOutcomes 2016;9: 39-47.

12. Rossini R, Tarantini G, Musumeci G, et al. A Multidisciplinary 13.Approach on the Perioperative Antithrombotic Management of Patients With Coronary Stents Undergoing Surgery Surgery After Stenting 2. acc.org/ jacc-journals-cmejacc: cardiovascular interventions vol. 11, no. 5, 2018 surgery after stenting 2 march 12, 2018:417- 34

13. Mahmoud KD, Sanon S, Habermann EB, et al. Perioperative cardiovascular risk of prior coronary stent implantation among patients undergoing noncardiac surgery. J Am CollCardiol 2016;67: 1038-49.

14. Valgimigli M, Bueno H, Byrne RA, et al. 2017 ESC focused update on dual antiplatelet therapy in coronary artery disease developed in collaboration with EACTS: The Task Force for dual antiplatelet therapy in coronary artery disease of the European Society of Cardiology (ESC) and of the European Association for Cardio-Thoracic Surgery (EACTS). Eur Heart J 2018;39:213-60.

15. Holmes DR Jr., Kereiakes DJ, Garg S, et al. Stent thrombosis. J Am CollCardiol 2010;56: 1357-65.
16. Mehran R, Baber U, Steg PG, et al. Cessation of dual antiplatelet treatment and cardiac events after percutaneous coronary intervention (PARIS): 2 year results from a prospective observational study. Lancet 2013:382:1714-22.

17. Kristensen SD, Knuuti J, Saraste A, et al. 2014 ESC/ESA guidelines on non-cardiac surgery: cardiovascular assessment and management: the Joint Task Force on non-cardiac surgery: cardiovascular assessment and management of the European Society of Cardiology (ESC) and the European Society of Anaesthesiology (ESA). Eur Heart J 2014;35:2383-431.

18. Holcomb CN, Graham LA, Richman JS, et al. The incremental risk of COronary stents on postoperative adverse events: a matched cohort study. Ann Surg 2016;263:924-30

19. Garg S, Sarno G, Garcia-Garcia HM, et al., ARTS-II Investigators. A new tool for the risk stratification of patients with complex coronary artery disease: the Clinical SYNTAX Score. CircCardiovascInterv 2010;3:317-26.

20. Parasca CA, Head SJ, Milojevic M, et al., SYNTAX Investigators. Incidence, characteristics, predictors, and outcomes of repeat revascularization after percutaneous coronary intervention and coronary artery bypass grafting: the SYNTAX trial at 5 years. J Am CollCardiollntv 2016;9: 2493-507.

21. Généreux P, Palmerini T, Caixeta A, et al. Quantification and impact of untreated coronary artery disease after percutaneous coronary intervention: the residual SYNTAX (Synergy Between PCI with Taxus and Cardiac Surgery) score. J Am CollCardiol 2012;59:2165-74.

22. Wijeysundera DN, Wijeysundera HC, Yun L, et al. Risk of elective major non cardiac surgery after coronary stent insertion: a population-based study. Circulation 2012;126:1355-62

23. Hawn MT, Graham LA, Richman JS, Itani KM, Henderson WG, Maddox TM. Risk of major adverse cardiac events following non cardiac surgery in patients with coronary stents. JAMA 2013;310: 1462-72.

24. Savonitto S, Caracciolo M, Cattaneo M, De Servi S. Management of patients with recently implanted coronary stents on dual antiplatelet therapy who need to undergo major surgery. J Thromb Haemost 2011;9:2133-42.

25. Sun JCJ, Whitlock $R$, Cheng J, et al. The effect of pre-operative aspirin on bleeding, transfusion, myocardial infarction and mortality in coronary artery bypass surgery: a systematic review of randomized and observational studies. Eur Heart J 2008; 29: 1057-71.

26. Burger W, Chemnitius J, Kneiss/ G, Rucker G. Low-dose aspirin for secondary cardiovascular prevention-cardiovascular risks after its perioperative withdrawal versus bleeding risks with its continuation. J Intern Med 2005; 257: 399-414.

27. Leong JY, Baker RA, Shah PJ, Cherian VK, Knight JL. Clopidogrel and bleeding after coronary artery bypass graft surgery. Ann ThoracSurg 2005, 80: 928-33.

28. Chassot PG, Delabays A, Spahn DR. Perioperative antiplatelet therapy: the case for continuing therapy in patients at risk of myocardial infarction. $B$ J Anaesth 2007; 99: 316-28.

29. Vivasa D, Roldan I, Ferrandis R, et al. Manejo perioperatorio y periprocedimiento del tratamiento antitrombótico: documento de consenso de SEC, SEDAR, SEACV, SECTCV, AEC, SECPRE, SEPD, SEGO, SEHH, SETH, SEMERGEN, SEMFYC, SEMG, SEMICYUC, SEMI, SEMES, SEPAR, SENEC, SEO, SEPA, SERVEI, SECOTY AEU. Revista Española de Cardiología. 2018. doi.org/10.1016/j.recesp.2018.01.001 
30. Vicenzi MN, Meislitzer T, Heitzinger B, Halaj M, Fleisher LA, Metzler H. Coronary artery stenting and non-cardiac surgery - a prospective outcome study. British Journal of Anaesthesia. 2006;96:686-93.

31. Graham MM, Sessler DI, Parlow JL, et al. Aspirin in patients with previous percutaneous coronary intervention undergoing noncardiac surgery. Ann Intern Med 2018;168:237-44

32. Merritt JC, Bhatt DL. The efficacy and safety of perioperative antiplatelet therapy. J Thromb Thrombolysis 2004;17:21-27.

33. Hansson EC, Jidéus L, Åberg B, et al. Coronary artery bypass grafting-related bleeding complications in patients treated with ticagrelor or clopidogrel: a nationwide study. Eur Heart J 2016;37: 189-97.

34. Wiviott SD, Braunwald E, McCabe CH, et al; TRITON-TIMI 38 Investigators. Prasugrel versus clopidogrel in patients with acute coronary syndromes. NEngl J Med 2007; 357: 2001-15.

35. Carrasco G, López B, Lema F. Coronarystents and non-cardiacsurgery: Current management strategies. Rev Med Chile 2011; 139: 787-793.

36. Capodanno D, Angiolillo DJ. Management of antiplatelet therapy in patients with coronary artery disease requiring cardiac and noncardiac surgery. Circulation 2013;128:2785-98.

37. Franchi F, Rollini F, Muñiz-Lozano A, Cho JR, Angiolillo DJ. Cangrelor: a review on pharmacology and clinical trial development. Expert Rev CardiovascTher 2013;11:1279-91

38. Angiolillo DJ, Schneider DJ, Bhatt DL, et al. Pharmacodynamic effects of cangrelor and clopidogrel: the platelet function substudy from the cangrelor versus standard therapy to achieve optimal management of platelet inhibition (CHAMPION) trials. J Thromb Thrombolysis 2012;34:44-55.

39. Bhatt DL, Stone GW, Mahaffey KW, et al., CHAMPION PHOENIX Investigators. Effect of platelet inhibition with cangrelor during PCl on ischemic events. N Engl J Med 2013;368:1303-13
40. Angiolillo DJ, Firstenberg MS, Price MJ, et al. Bridging antiplatelet therapy with cangrelor un patients undergoing cardiac surgery. A randomized control trial. JAMA 2012;307:265-74.

41. Angiolillo DJ, Rollini F, Storey RF, et al. International expert consensus on switching platelet P2Y12 receptor-inhibiting therapies. Circulation 2017; 136:1955-75.

42. Alshawabkeh $L I$, Prasad A, Lenkovsky $F$, et al. Outcomes of a preoperative "bridging" strategy with glycoprotein Ilb/llla inhibitors to prevent perioperative stent thrombosis in patients with drug-eluting stents who undergo surgery necessitating interruption of thienopyridine administration. Eurolntervention. 2013; 9:204-11.

43. Pickett AM, Taylor DA, Ackman ML. Prolonged infusion of eptifibatide as bridge therapy between bare-metal stent insertion and cardiovascular surgery: casereport and review of theliterature. Pharmacotherapy 2010;30:127-133e.

44. Savonitto S, Urbano M, Caracciolo M, et al. Urgentsurgery in patients with a recently implanted coronary drug-eluting stent: a phase ll study of bridging antiplatelet therapy with tirofiban during temporary withdrawal of clopidogrel. Br J Anaesth 2010; 104: 285-91.

45. Rodriguez AE, Granada JF, AlemparteMR, et al.Oral Rapamycin After Coronary Bare-Metal Stent Implantation to Prevent Restenosis: The Prospective, Randomized Oral Rapamycin in Argentina (ORAR II) Study.Journal of the American College of Cardiology. Volume 47, Issue 8, 18 April 2006, 1522-1529.

46. Rodriguez AE,Maree A,Tarragona S, et al.Percutaneous coronary intervention with oral sirolimus andbare metal stents has comparable safety and efficacy totreatment with drug eluting stents, but with significant costsaving long-term follow-up results from the randomised, controlled ORAR III (Oral Rapamycin in ARgentina) study.Eurolntervention 2009;5:255-264 published online ahead of print May 2009. 


\section{ANEX01}

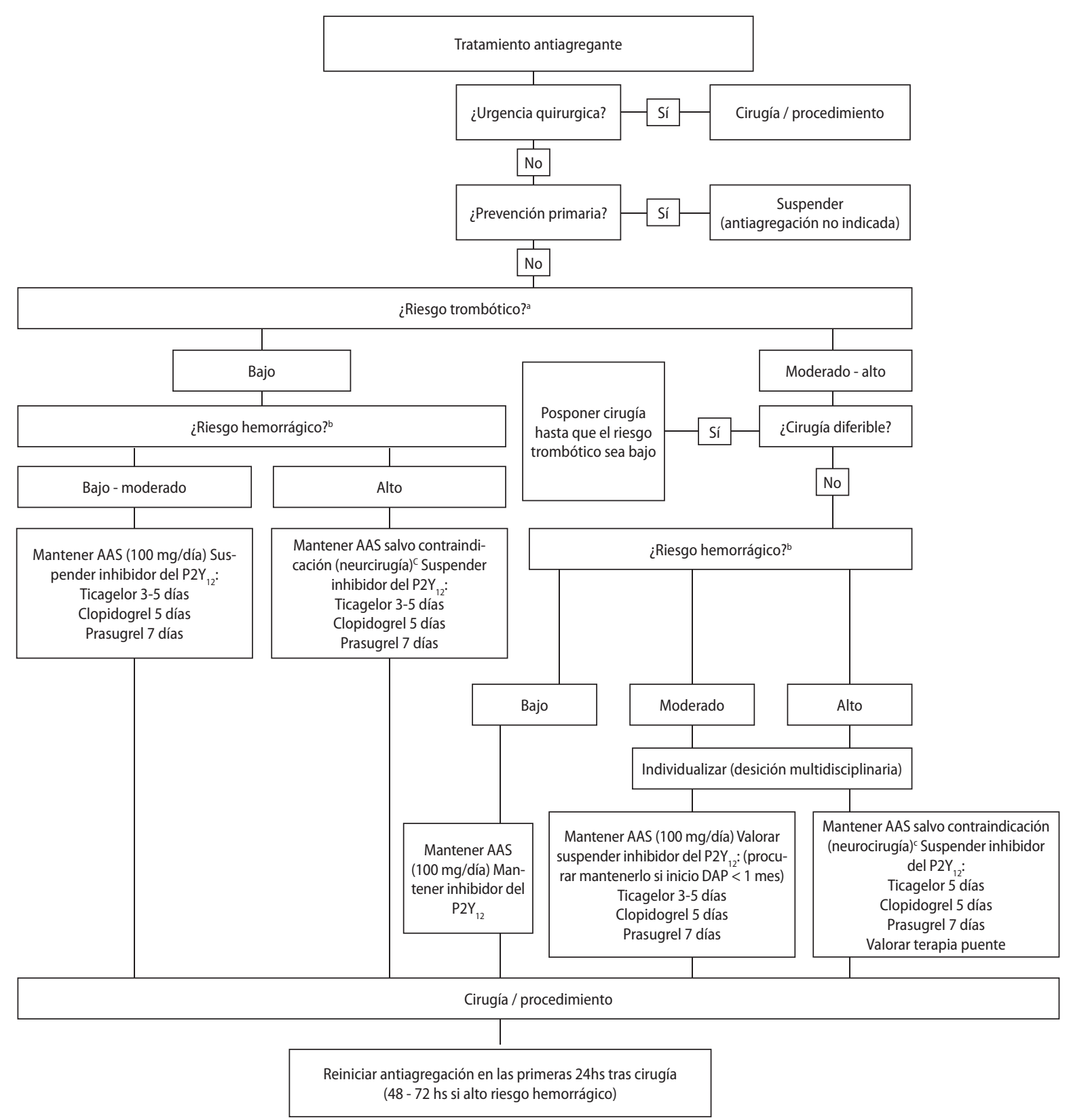

Anexo 1. Recomendaciones para la suspensión y reinicio de antiagregación plaquetaria en función del tipo de intervención quirúrgica y los riesgos trombóticos y hemorrágicos (tomado de ref. № 28.). AAS: ácido acetilsalicílico. DAP: doble antiagregación plaquetaria. a. Clasificación del riesgo trombótico. b. Clasificación del riesgo hemorrágico. c. De ser necesario, suspender el AAS 3 días antes de la intervención.

TABLA 1. Riesgo trombótico en pacientes con stent coronario. (basado en refs. № 11, 45 y 46)..

\begin{tabular}{|l|c|c|c|c|c|c|}
\hline & \multicolumn{3}{|c|}{$\begin{array}{c}\text { Con características clínicas o angiográficas } \\
\text { de riesgo isquémico aumentadas }\end{array}$} & \multicolumn{2}{c|}{$\begin{array}{c}\text { Sin características clínicas o angiográficas } \\
\text { de riesgo isquémico aumentadas }\end{array}$} \\
\hline $\begin{array}{l}\text { Tiempo desde implante } \\
\text { de stent a la Cx }\end{array}$ & $\begin{array}{c}\text { Stent no } \\
\text { farmacológico }\end{array}$ & $\begin{array}{c}\text { Stent farmacológico } \\
\text { 1a generación }\end{array}$ & $\begin{array}{c}\text { Stent farmacológico } \\
2 \text { da generación }\end{array}$ & $\begin{array}{c}\text { Stent no } \\
\text { farmacológico }\end{array}$ & $\begin{array}{c}\text { Stent farmacológico } \\
\text { la generación }\end{array}$ & $\begin{array}{c}\text { Stent farmacológico } \\
2 \text { da generación }\end{array}$ \\
\hline$<1$ mes & Alto & Alto & Alto & Alto & Alto & Alto \\
\hline $1-3$ meses & intermedio & Alto & Alto & Intermedio & Alto & intermedio \\
\hline 4-6 meses & Bajo & Alto & Intermedio/alto & Bajo & & Bajo/intermedio \\
\hline 6-12 meses & Bajo & intermedio & intermedio & Bajo & intermedio & Bajo \\
\hline$>12$ meses & Bajo & bajo & Bajo & Bajo & Bajo & Bajo \\
\hline
\end{tabular}




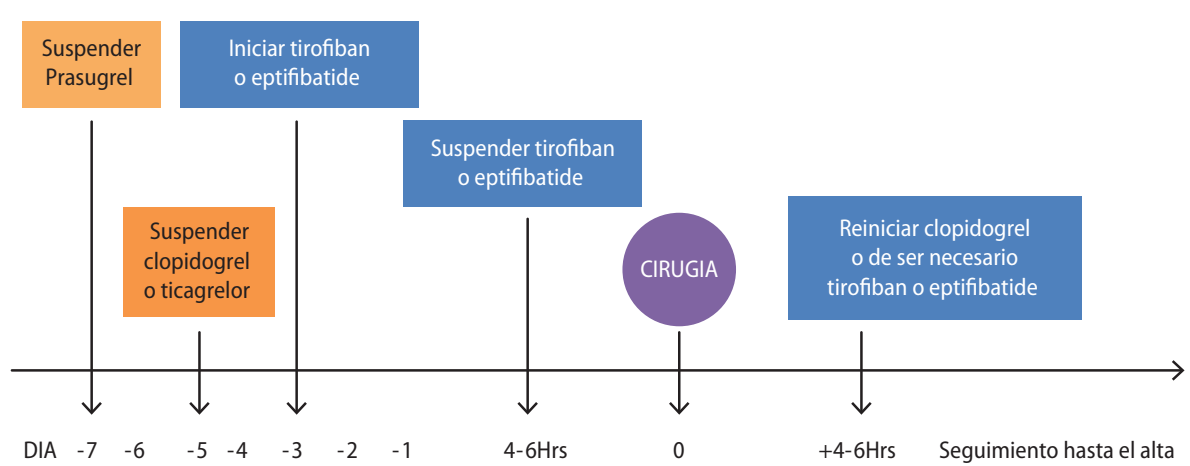

Figura 1. Terapia puente con tirofiban o eptifibatide. (adaptado de Rossini et al)

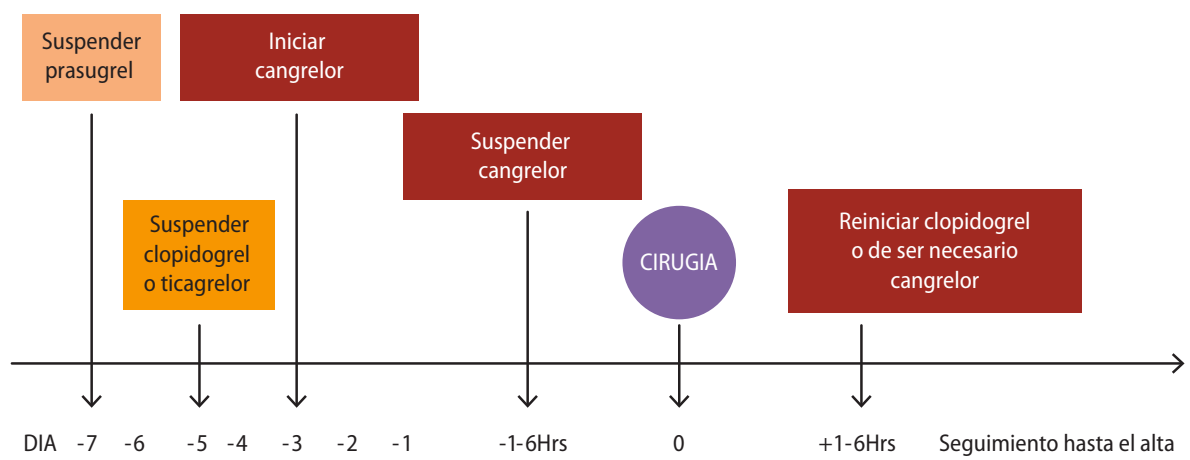

Figura 2. Terapia puente con cangrelor (adaptado de Rossini et al).

TABLA 2. Caracteristicas clínicas y angiográficas que incrementan el riesgo isquémico en pacientes tratados con stents coronarios (basado en 11).

\begin{tabular}{|c|c|}
\hline Caracteristicas clínicos de riesgo & $\begin{array}{l}\text { ICP indicada en contexto de SCA } \\
\text { Múltiples IM anteriores } \\
\text { Stent trombosis previa con TAP adecuada } \\
\text { FEVI }<35 \% \\
\text { Enfermedad renal crónica } \\
\text { Diabetes mellitus }\end{array}$ \\
\hline Caracteristicas angiograficos de riesgo & $\begin{array}{l}\text { Stents largos o múltiples (al menos } 3 \text { stents implantados o } 3 \text { lesiones tratadas o longitud total del stent }>60 \text { mm) } \\
\text { Stents superpuestos } \\
\text { Pequeño diámetro del stent (<2.5 mm) } \\
\text { Lesiones de bifurcación (con } 2 \text { stents implantados) } \\
\text { Enfermedad coronaria extensa } \\
\text { Revascularización incompleta } \\
\text { Tratamiento de la oclusión total crónica }\end{array}$ \\
\hline
\end{tabular}

